\title{
Autofluorescencia de retina en la evaluación del edema macullar diabético
}

\section{Fundus autofluorescence in the assessment of diabetic macular edema}

\author{
Sergio E. Hernández-Da Mota ${ }^{1 *}$, Ernesto Rodríguez-Ayala², Jorge J. Fromow-Guerra ${ }^{3}$ y \\ Enrique A. Roig Melo-Granados ${ }^{4}$
}

${ }^{1}$ Servicio de Retina, Clínica David, Unidad Oftalmológica, Morelia, Mich.; ${ }^{2}$ Facultad de Ciencias de la Salud, Universidad Anáhuac Norte, Huixquilucan, Edo. de México; ${ }^{3}$ Servicio de Retina, Hospital Dr. Luis Sánchez Bulnes, Asociación para Evitar la Ceguera en México, UNAM, Ciudad de México; ${ }^{4}$ Servicio de Retina, Hospital Civil de Guadalajara, Guadalajara, Jal. México

\section{Resumen}

Objetivo: Evaluar la asociación entre la autofluorescencia de retina $(A F)$ y las variables estructurales de la tomografía de coherencia óptica (OCT) y de la angiografía con fluoresceína (FA) en pacientes con edema macular diabético (EMD). Métodos: El diseño del estudio fue una serie de casos no comparativa. A 37 ojos de 20 pacientes con EMD se les realizó la determinación de la agudeza visual mejor corregida y la sensibilidad al contraste, y la determinación del grosor foveal central, grosor promedio macular (GPM), volumen macular (VM) y patrones de EMD mediante OCT de dominio espectral (OCT-DE). Se tomaron imágenes de AF y de FA. Se evaluó el patrón de AF foveal (disminuida, normal, aumentada unifocal y aumentada multifocal). Se efectuó prueba exacta de Fisher, variabilidad interobservador y prueba de Kruskall-Wallis como análisis estadístico. Resultados: Se encontró una diferencia estadísticamente significativa entre los cuatro patrones de AF en el VM ( $p=0.03)$ y en el GPM ( $p=0.014)$. El análisis post hoc mostró, en el VM, una diferencia estadísticamente significativa entre el patrón de AF disminuida y el de AF normal ( $p<0.05)$, así como entre el patrón de AF disminuida y el de AF aumentada multifocal ( $p<0.05)$. La fuga de la red capilar perifoveal por FA se presentó en mayor proporción en los patrones de AF aumentada tanto uni como multifocal $(p=0.032)$. Conclusiones: Existen cambios en la AF con cámara de flash, en pacientes con EMD asociados con el GPM, el VM y a la fuga de red capilar perifoveal. Es importante establecer el valor de la AF en la evaluación del EMD.

Palabras clave: Retinopatía diabética. Edema macular diabético. Autofluorescencia de retina. Tomografía de coherencia óptica. Angiografía con fluoresceína.

\section{Abstract}

Objective: To assess the correlation between fundus autofluorescence (FAF) and optical coherence tomography (OCT) and fluorescein angiography (FA) parameters in patients with diabetic macular edema (DME). Methods: Study design: non-comparative case series. Thirty-seven eyes of 20 patients with DME were included. Patients underwent best-corrected visual acuity and contrast sensitivity determination, assessment of central subfield thickness (CST), macular volume (MV), and macular cube average thickness (MCAT) by spectral-domain OCT, as well as FAF and FA imaging. SD-OCT EMD and foveal

\section{Correspondencia:}

*Sergio E. Hernández-Da Mota

Blvd. García de León 598-2

Col. Nueva Chapultepec

Fecha de recepción: 21-02-2019

Fecha de aceptación: 24-02-2020

C.P. 58280, Morelia, Michoacán, México

E-mail: tolodamota@yahoo.com.mx

DOI: 10.24875/RMO.M20000114

0187-4519/@ 2020 Sociedad Mexicana de Oftalmología. Publicado por Permanyer. Este es un artículo open access bajo la licencia CC BY-NC-ND (http://creativecommons.org/licenses/by-nc-nd/4.0/).
Disponible en internet: 01-07-2020 Rev Mex Oftalmol. 2020;94(4):151-158 www.rmo.com.mx 
FAF patterns (decreased, normal, increased single-spot, and increased multiple-spot) were evaluated. Fisher exact test, interobserver variability, and Kruskall-Wallis test were used for statistical analysis. Results: $A$ statistically significant difference was observed between the four FAF foveal patterns in the MV ( $p=0.03)$, and in the MCAT groups $(p=0.01)$. Post-hoc analysis with the U-Mann-Whitney test showed a statistically significant difference between decreased FAF and normal FAF patterns, and between decreased FAF and increased multiple-spot FAF patterns $(p<0.05)$ in the MV and MCAT groups. Foveal capillary leakage by FA was observed in a higher proportion of eyes in the increased single-spot and multiple-spot FAF patterns $(p=0.03)$. Conclusions: Flash camera FAF shows changes regarding MV, MCAT, and the presence of foveal capillary leakage in patients with DME. It is relevant to establish the value of FAF in the assessment of DME.

Key words: Diabetic retinopathy. Diabetic macular edema. Fundus autofluorescence. Optical coherence tomography. Fluorescein angiography.

\section{Introducción}

El edema macular diabético (EMD) es la principal causa de baja visual en el paciente diabético ${ }^{1-3}$. Constituye una de las alteraciones presentes en la retinopatía diabética, y es causa importante de discapacidad visual en México y el mundo 4 .

La evaluación integral del EMD, sobre todo en la era del paradigma multimodal diagnóstico de la patología vitreorretiniana, incluye la evaluación de la función visual, la biomicroscopía del segmento posterior, la angiografía con fluoresceína (FA) y la tomografía de coherencia óptica (OCT) $)^{5,6}$.

Un estudio de más reciente advenimiento y aplicación en la evaluación del EMD es la autofluorescencia (AF) de retina. La AF, empero, ha sido relativamente poco explorada en el EMD, tanto en el diagnóstico como en el seguimiento de los pacientes tratados con esta entidad, sobre todo con los sistemas de obtención de imágenes de AF con cámara de flash. Los sistemas de cámara de flash tienen la ventaja de ser más accesibles por su costo, comparados con los sistemas de láser de barrido confocal ${ }^{5-16}$. Varios autores han explorado la utilidad de la $\mathrm{AF}$ en la evaluación y respuesta a tratamientos del EMD ${ }^{6}$. Vujosevic, et al. ${ }^{13}$ encontraron una correlación entre la $A F$, empleando un sistema de obtención de imágenes mediante láser de barrido confocal, y variables funcionales como la sensibilidad obtenida mediante microperimetría. Existe, por ende, la necesidad de evaluar la asociación entre la AF con los sistemas basados en cámara de flash y parámetros existentes en otras modalidades de imagen como la OCT, así como variables para evaluar a la función retiniana, con la finalidad de poder determinar su papel en la evaluación del EMD.

\section{Objetivo}

El propósito de este estudio fue el de evaluar la asociación entre las imágenes de AF obtenidas mediante un sistema de cámara de flash y las variables estructurales obtenidas mediante OCT de dominio espectral (OCT-DE), pruebas funcionales y de FA retinianas, en pacientes con EMD.

\section{Métodos}

El diseño del estudio fue observacional, prospectivo, transversal y analítico. Todos los pacientes fueron reclutados del Servicio de Retina de la Clínica David, Unidad Oftalmológica de la Ciudad de Morelia, Michoacán, México, de octubre de 2017 a mayo de 2018. Se incluyeron pacientes con presencia de EMD detectado clínicamente o por OCT.

Se excluyeron pacientes con presencia de catarata significativa (determinada por la presencia de la calidad de la imagen de la OCT con una fuerza de la señal menor de 6), componente traccional del edema determinado mediante la OCT, otras patologías maculares (incluida la degeneración macular relacionada a la edad y datos de oclusión venosa retiniana), tratamiento previo con terapia antiangiogénica intravítrea, tratamiento previo con láser selectivo macular.

A todos los pacientes se les realizó un examen oftalmológico completo que incluyó determinación de la agudeza visual mejor corregida (AVMC), sensibilidad al contraste (SC) y examen biomicroscópico del segmento anterior y posterior. Se les realizaron también estudios de AF con cámara de flash, FA, OCT-DS y fotografía clínica del segmento posterior.

Se obtuvo consentimiento informado de todos los pacientes incluidos y el estudio fue aprobado por el Comité de ética de la Clínica David, Unidad Oftalmológica. Se acató el reglamento de la Ley General de Salud en Materia de Investigación para la Salud en lo referente a los aspectos éticos de la investigación en seres humanos.

El estudio se realizó también de acuerdo con el código de Nuremberg y a los principios de la Declaración 
de Helsinki y siguiendo los lineamientos establecidos por las normas oficiales mexicanas NOM 04 y NOM 012 de la Secretaría de Salud.

\section{Procedimientos realizados en los pacientes}

Agudeza visual mejor corregida (AVMC). La AVMC lejana para cada ojo se midió utilizando el protocolo estándar del Early Treatment Diabetic Retinopathy Study (ETDRS) a una distancia de 4 metros con la cartilla modificada ETDRS (Precision Vision, La Salle, IL, EE. UU.). La AVMC se registró como el número total de letras leídas correctamente en cada ojo por separado.

Sensibilidad al contraste (SC). La SC se midió en todos los pacientes empleando la cartilla de SC de Hamilton-Veale (Hamilton Veale, Canterbury, Nueva Zelanda). Se registró la SC como el número total de pares de letras leídas correctamente a 1 metro de distancia en cada ojo por separado.

Análisis cualitativo de la AF. La imágenes de AF se obtuvieron con una cámara de fondo (cámara de fondo modelo Visucam NM/FA, Carl Zeiss Meditec, Oberkochen, Alemania). Se empleó una longitud de onda de 510-580 nm para la excitación, mientras que la luz emitida se detectó por encima de $640 \mathrm{~nm}$ debido a un filtro de barrera. Se tomaron varias imágenes y se descartaron las que tenían artefactos, como la presencia de sombras o visualización deficiente. Las imágenes digitales seleccionadas se guardaron en la computadora para su posterior análisis, clasificación y procesamiento. Las tomas fueron mejoradas con el editor automático del software de la cámara Visucam. Se diseñó una clasificación para los patrones de AF, modificando a la publicada por Vujosevic, et al. ${ }^{13}$. De esta manera, las imágenes de AF se clasificaron de acuerdo con diferentes patrones de $A F$ en el área foveal: $A F$ disminuida, AF normal, AF aumentada unifocal y AF aumentada multifocal (Fig. 1). La clasificación de las imágenes de AF se realizó de forma independiente por dos evaluadores expertos de manera enmascarada.

Fotografía de fondo y FA. En todos los pacientes, después de la dilatación de la pupila, un fotógrafo entrenado con la misma cámara de fondo (equipo modelo Visucam NM/FA fundus camera, Carl Zeiss Meditec, Oberkochen, Alemania) tomó fotografías del polo posterior a color y de FA. Las imágenes de FA de la mácula se clasificaron en función de la presencia o ausencia de isquemia y fuga del colorante. Las tomas fotográficas de la AF se realizaron primero que los otros estudios de imagen.

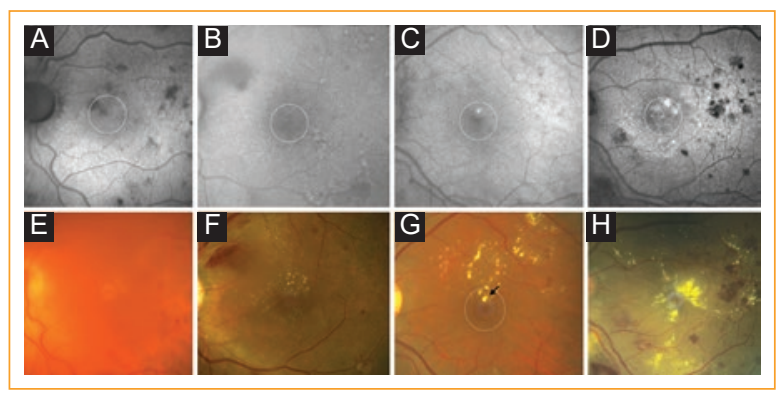

Figura 1. Patrones de autofluorescencia (AF) en el área foveal. A: AF disminuida; B: AF normal; C: AF aumentada unifocal; D: AF aumentada multifocal. La imagen de cada patrón va acompañada en la parte inferior por su correspondiente imagen clínica.

Tomografía de coherencia óptica (OCT). Se realizó un escaneo o barrido de OCT-DE (equipo modelo Cirrus 5000 OCT-DE; Carl Zeiss Meditec, Oberkochen, Alemania) del área macular. El protocolo de barrido empleado para este estudio fue el grosor macular, calculado de acuerdo con el programa de cubo macular 512 $x$ 128. Se realizó el análisis de la imagen estructural del reporte PDF. Para este estudio, el grosor de la retina en el milímetro central se usó como medida de la OCT del grosor foveal central (GFC). Se midieron el volumen macular (VM) en $\mathrm{mm}^{3}$ y el grosor promedio macular (GPM) en micras $(\mu \mathrm{m})$. Estas imágenes se guardaron también en el disco duro para su análisis ulterior. Se clasificó el EMD en cuatro patrones de acuerdo con la clasificación descrita por Otani, et al. ${ }^{17}$ : focal, difuso quístico, difuso espongiforme (no quístico) y difuso con desprendimiento neurorretiniano seroso subfoveal.

Análisis estadístico. Se realizó estadística de tipo descriptivo y posteriormente se aplicó la prueba de normalidad de Kolmogorov-Smirnov para evaluar la normalidad en la distribución de los datos. Se empleó la prueba de Kruskall-Wallis para comparar grupos y la U de MannWhitney como prueba post hoc. Se empleó la prueba exacta de Fisher para comparar frecuencias esperadas pequeñas. Se determinó el coeficiente $\kappa$ para evaluar variabilidad interobservador. El nivel mínimo de significancia que se consideró fue de $p<0.05$. Se empleó como software estadístico el programa SPSS para Mac (IBM Corp. Released 2012. IBM SPSS Statistics for Mac, Version 21.0. Armonk, NY: IBM Corp).

\section{Resultados}

En este estudio se incluyeron 37 ojos de 20 pacientes. Las edades de los pacientes tuvieron un rango de entre 
Tabla 1. Características de los pacientes incluidos en el estudio

\begin{tabular}{|c|c|c|c|}
\hline \multicolumn{2}{|l|}{ Sexo de los pacientes, n (\%) } & \multicolumn{2}{|c|}{ Patrón de AF, n (\%) [IC 95]* } \\
\hline Masculino & $12(60)$ & Disminuido & $5(13)[3-27]$ \\
\hline Femenino & $8(40)$ & Normal & $15(40)$ [23-59] \\
\hline Edad, años & & Aumentado unifocal & $6(17)[3-30]$ \\
\hline Promedio $\pm \mathrm{DE}$ & $62.8 \pm 7.2$ & Aumentado multifocal & $11(30)[13-47]$ \\
\hline Rango (años) & $53-79$ & \multirow{3}{*}{\multicolumn{2}{|c|}{ Presencia de fuga de la red capilar por FA, $n(\%)^{*}$}} \\
\hline Tiempo de evolución de la DM (años) & $11 \pm 5.6$ & & \\
\hline HAS asociada (\% de pacientes) & 80 & & \\
\hline AVMC, número de letras & $31.5 \pm 16$ & Ausente & $8(30.8)$ \\
\hline SC, número de pares de letras & $7 \pm 3.5$ & Presente & $19(69.2)$ \\
\hline \multicolumn{4}{|l|}{ Patrones de EMD por OCT, n (\%)* } \\
\hline Focal & & & $7(17.9)$ \\
\hline Difuso quístico & & & $6(15.4)$ \\
\hline Difuso no quístico (espongiforme) & & & $20(51.3)$ \\
\hline \multicolumn{2}{|c|}{ Difuso con desprendimiento neurorretiniano seroso subfoveal } & & $6(15.4)$ \\
\hline
\end{tabular}

AF: autofluorescencia de retina; AVMC: agudeza visual mejor corregida; FA: angiografía con fluoresceína; EMD: edema macular diabético; HAS: hipertensión arterial sistémica; OCT: tomografía de coherencia óptica; SC: sensibilidad al contraste.

53 y 79 años (promedio, $62.8 \pm 7.2$ ). Fueron de sexo masculino 12 pacientes y del femenino 8 pacientes. Las medias de la AVMC y de la SC fueron de $31.5 \pm 16$ letras y $7.04 \pm 3.5$ pares de letras, respectivamente.

Las medias del GFC, VM y GPM de los pacientes fue de $356.3 \mu \mathrm{m} \pm 123.5,10.9 \mathrm{~mm}^{3} \pm 3$ y $363.9 \mu \mathrm{m} \pm$ 93.9, respectivamente. Los patrones de AF fueron: disminuida (13\%), normal $(40 \%)$, aumentada unifocal (17\%) y aumentada multifocal (30\%) (Fig. 1).

Se observaron los siguientes patrones de edema por OCT: focal (17.9\%), difuso quístico (15.4\%), difuso no quístico o espongiforme (51.3\%) y difuso con desprendimiento neurorretiniano seroso subfoveal (15.4\%) (Tabla 1).

La confiabilidad interobservador entre los dos investigadores para la clasificación de la AF (grados 1 a 4) se evaluó con un coeficiente $\kappa$ de $0.906(p=0.000)$.

El análisis con ANOVA no paramétrico o prueba de Kruskall-Wallis mostró una diferencia estadísticamente significativa entre los cuatro patrones de AF en el caso del VM $(p=0.03)$ y del GPM $(p=0.014)$ (Tabla 2).

Por otro lado, el análisis post hoc con la prueba $U$ de Mann Whitney, en el caso del VM, mostró que hubo diferencias estadísticamente significativas entre el grupo de AF disminuida y el de AF normal $(p=0.024)$ y entre el de AF disminuida y el de AF aumentada multifocal ( $p=0.022)$.

En el caso del GPM, se encontró diferencia estadísticamente significativa entre el patrón de AF disminuida y el de AF normal $(p=0.012)$, así como entre el patrón de AF disminuida y el de AF aumentada multifocal $(p=0.003)$.

No hubo diferencias estadísticamente significativa entre los patrones de AF y los patrones de EMD por OCT $(p=0.21)$.

Por el contrario, la fuga de la red capilar perifoveal por FA se presentó en mayor proporción en los patrones de AF aumentada tanto uni como multifocal $(p=$ 0.032) (Tabla 3). La figura 2 muestra un caso de AF aumentada unifocal y muestra la correlación con sus imágenes multimodales: clínica, de OCT-DE y de FA.

\section{Discusión}

Las imágenes de AF son una herramienta de gran utilidad en el diagnóstico y en el seguimiento de diferentes enfermedades retinianas, como los trastornos retinianos hereditarios como la enfermedad de Stargardt, la degeneración macular relacionada a la edad y el EMD?

En el EMD, si bien la AF ha demostrado beneficio, su uso ha estado más limitado y ha sido poco difundido 
Tabla 2. Diferencias entre las variables evaluadas de tomografía de coherencia óptica (OCT) (grosor foveal central [GFC], volumen promedio macular [VM], grosor promedio macular [GPM]) entre los cuatro patrones de autofluorescencia de retina (AF) encontrados. Se comparan también variables funcionales (agudeza visual mejor corregida [AVMC] y sensibilidad al contraste [SC])

\begin{tabular}{|l|c|c|c|c|c|}
\hline \multicolumn{7}{|c|}{} \\
\hline & AF disminuida & AF normal & AF aumentada unifocal & AF aumentada multifocal & p (Kruskall-Wallis) \\
\hline AVMC (n de letras) & $25 \pm 13.2$ & $28.4 \pm 17.3$ & $20 \pm 14.1$ & $25.6 \pm 18.4$ & 0.70 \\
\hline SC (n de pares de letras) & $6 \pm 3.1$ & $7.2 \pm 3.5$ & $5.5 \pm 6.3$ & $5.8 \pm 3$ & 0.70 \\
\hline GFC $(\mu)$ & $474 \pm 144.2$ & $385.2 \pm 159.7$ & $463.5 \pm 177.5$ & $400 \pm 142$ & 0.31 \\
\hline VM $\left(\mathrm{mm}^{3}\right)$ & $8.8 \pm 2.2$ & $10.9 \pm 2.7$ & $13.6 \pm 0.7$ & $13.5 \pm 2.62$ & 0.03 \\
\hline GPM $(\mu)$ & $437 \pm 35.3$ & $341.8 \pm 29.5$ & $378.5 \pm 21.9$ & $374.1 \pm 73.8$ & 0.01
\end{tabular}

Tabla 3. Diferencias entre los diferentes patrones de EMD por OCT y presencia o ausencia de fuga de red capilar perifoveal evaluada mediante el estudio de FA (angiografía con fluoresceína)

\begin{tabular}{|c|c|c|c|c|c|}
\hline \multicolumn{6}{|c|}{ Pa trón AF foveal } \\
\hline & $\begin{array}{c}\text { AF disminuida } \\
n(\%)\end{array}$ & $\begin{array}{c}\text { AF normal } \\
n(\%)\end{array}$ & $\begin{array}{l}\text { AF aumentada } \\
\text { unifocal } n(\%)\end{array}$ & $\begin{array}{l}\text { AF aumentada } \\
\text { multifocal } n(\%)\end{array}$ & $\begin{array}{l}\text { p (prueba exacta } \\
\text { de Fisher) }\end{array}$ \\
\hline $\begin{array}{l}\text { Patrón de OCT } \\
\text { Focal } \\
\text { Quístico } \\
\text { Espongiforme } \\
\text { DSRNS }\end{array}$ & $\begin{array}{l}- \\
2(33.3) \\
3(16.6) \\
1(16.6)\end{array}$ & $\begin{array}{l}6(85.7) \\
1(16.6) \\
6(33.3) \\
1(16.6)\end{array}$ & $\begin{array}{l}1(14.3) \\
2(33.3) \\
2(11.1) \\
-\end{array}$ & $\begin{array}{l}- \\
1(16.6) \\
7(38.8) \\
4(66.6)\end{array}$ & $p=0.21$ \\
\hline $\begin{array}{l}\text { Presencia de fuga } \\
\text { por FA } \\
\text { Fuga red capilar perifoveal } \\
\text { No fuga red capilar perifoveal }\end{array}$ & $\begin{array}{l}3(17.6) \\
1(12.5)\end{array}$ & $\begin{array}{l}5(29.4) \\
6(75)\end{array}$ & $\begin{array}{c}4(23.5) \\
-\end{array}$ & $\begin{array}{l}5(29.4) \\
1(12.5)\end{array}$ & $p=0.034$ \\
\hline
\end{tabular}

en la comunidad de médicos dedicados al tratamiento de la patología macular ${ }^{5,6,10-16,18,19}$.

Diversos autores ${ }^{11,13,16}$ han descrito diferentes patrones de cambios en la AF en los pacientes que padecen de EMD. Pece, et al. ${ }^{16}$ describieron dos tipos de patrones de AF en el EMD: el tipo 1, al que denominaron multilobulado, caracterizado por la presencia de múltiples áreas de AF aumentada, similar al de AF aumentada multifocal de nuestra clasificación; el tipo 2, también llamado unilobulado, que se corresponde con la presencia de un gran quiste en la imagen de OCT, y el tipo 3 o mixto, con características combinadas de los tipos 1 y 2 .

Vujosevic, et al..$^{13}$ describieron una clasificación con los siguientes patrones de AF dentro del área foveal: normal, AF aumentada unifocal y AF aumentada multifocal.
Para la obtención de las imágenes de AF emplearon un sistema de láser de barrido confocal. Los casos de imágenes que se apreciaron con AF disminuida los consideraron como producto del bloqueo que producen los pigmentos maculares sobre la AF y los clasificaron como parte del patrón normal de AF.

En la AF obtenida mediante los sistemas de cámara de flash, como el empleado en nuestro estudio, tanto la excitación como la emisión de la señal de AF ocurren en una longitud de onda mayor, lo que se traduce en un aspecto de menor densidad de los pigmentos maculares en la imagen obtenida.

Hubo casos en nuestro estudio, donde apreciamos AF más disminuida, sobre todo en la periferia de la fóvea y en la zona limítrofe entre la fóvea y la parafóvea, que no consideramos como parte del patrón de AF normal. 


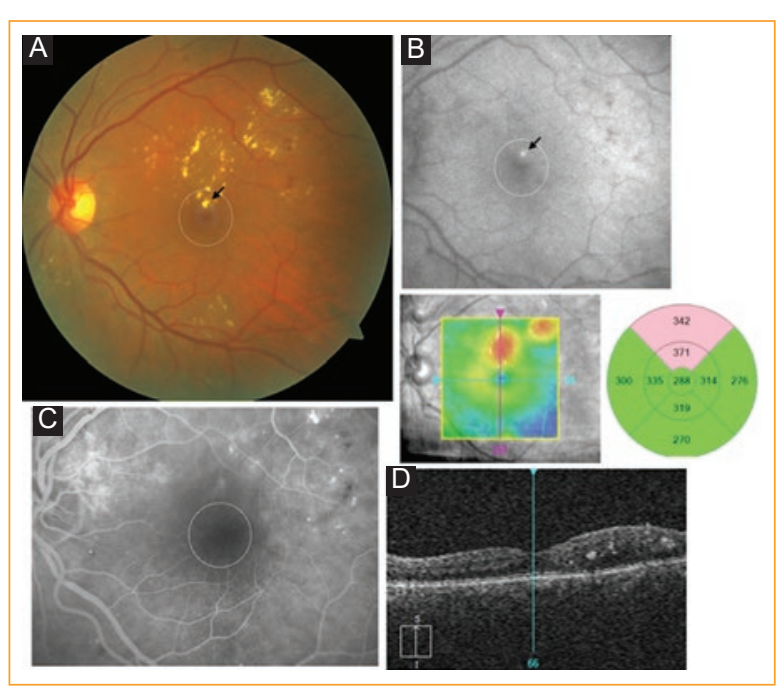

Figura 2. Edema macular diabético con presencia de patrón foveal de autofluorescencia (AF) aumentada unifocal. A: Fotografía clínica del polo posterior (la flecha señala exudado duro de aspecto más refringente). B: Imagen de AF (la flecha indica la AF aumentada unifocal que se corresponde con el exudado de aspecto refringente de la foto clínica). C: Imagen de FA. D: Imagen infrarroja con montaje de grosor en color de la tomografía de coherencia óptica. Se aprecia zona focal de engrosamiento macular que se corresponde con el foco de AF aumentada de la imagen $B$.

Las características de aspecto irregular, paracentral, que se correspondían con zonas de engrosamiento focal en la OCT, en estos casos de AF disminuida, nos hicieron pensar que probablemente no estaban dados por la presencia de pigmentos maculares. Por esta razón, decidimos modificar la clasificación descrita por Vujosevic, et al. ${ }^{13}$ y agregamos un nuevo patrón: el de AF disminuida. Hipotetizamos que una AF más reducida en este patrón podría deberse no a los pigmentos maculares, sino a la presencia de un aumento del grosor en la periferia del área foveal, debiéndose considerar como una categoría o patrón separado de la AF foveal.

Debido a que la AF disminuida está relacionada con el aumento del grosor de la retina en el área parafoveal, Yoshitake, et al. ${ }^{18}$ plantearon la hipótesis de que la AF disminuida en estos ojos refleja la autofluorescencia del epitelio pigmentario de la retina (EPR) bloqueada por el parénquima retiniano edematoso, principalmente en el patrón espongiforme del EMD. Lo anteriormente expuesto, pudiera explicar el hecho de que el GPM se encontró mayor en el grupo de AF disminuida que en el resto de los grupos.

Por el contrario, en el patrón quístico del EMD, los quistes que se ubican en las capas plexiforme externa y nuclear interna en el área foveal, sobre todo en el centro de la fóvea (zona que es de menor grosor comparada con la retina circundante), donde hay un acúmulo máximo de pigmento lúteo y en donde producen una imagen de AF aumentada. Los quistes desplazan al pigmento lúteo y evitan el bloqueo normal de la señal de AF foveal a nivel de cada uno de ellos, dando los patrones de AF aumentada uni y multifocal.

Chung, et al. ${ }^{11}$ describieron otro sistema de clasificación de la AF, pero a nivel foveolar exclusivamente: los ojos sin AF aumentada en la foveola fueron clasificados como de grado 1, los ojos con AF aumentada de menos de la mitad del área foveolar, como de grado 2, los ojos con AF aumentada entre la mitad y tres cuartos del área foveolar, como de grado 3, y, por último, cuando la AF aumentada ocupaba la totalidad del área foveolar, como de grado 4 .

Estas clasificaciones de los diferentes patrones de AF, sobre todo con los sistemas confocales, han servido para el diseño de estudios en los cuales se ha evaluado la correlación entre AF y variables anatomofuncionales retinianas ${ }^{10-16,18}$.

Reznicek, et al..$^{15}$, por ejemplo, evaluaron el papel de la $A F$ de longitud de onda verde en el EMD. Sus resultados indicaron una débil correlación de la intensidad de la AF con el GFC, la integridad de la capa elipsoide retiniana y el grosor de la capa-complejo EPR-elipsoide en pacientes diabéticos con o sin EMD. En nuestro estudio no encontramos asociación con el GFC, pero sí con el VM y el GPM.

Pece, et al. $^{16}$ evaluaron las imágenes de AF en pacientes con retinopatía diabética y EMD de aspecto quístico y su correlación con los hallazgos de FA y OCT. Encontraron que los hallazgos de la FA y el OCT tuvieron una correlación positiva con el aumento quístico de AF. En nuestro estudio, encontramos también una mayor proporción de casos con fuga de red capilar perifoveal en los ojos con patrones de AF aumentada, tanto uni como multifocal.

Chung, et al. ${ }^{11}$ describieron que la AF se correlacionó bien con datos cuantitativos. Evaluaron la capacidad visual y las imágenes de AF y de OCT-DE que se obtuvieron antes y después de la inyección de bevacizumab intravítreo para el tratamiento del EMD. Describieron que el EMD mostró una correlación significativa con el aumento de AF de la foveola, sobre todo en lo referente a biomarcadores de imagen, como disrupción de las capas externas de la retina como la membrana limitante externa. Al igual que Reznicek, et al. y a diferencia también de nuestro estudio, encontraron 
una correlación positiva entre el GFC y el grado de AF retiniana.

Chung, et al. ${ }^{11}$ también encontraron que la AF foveolar fue 4.2 veces más probable que ocurriera en EMD con presencia de quistes intrarretinianos. Llegaron a la conclusión de que la asociación entre los parámetros de AF y OCT-DE, así como la visión en pacientes con EMD, podría predecir el restablecimiento de la integridad del fotorreceptor y la posterior recuperación visual, especialmente en casos de EMD grave en los que la integridad del fotorreceptor antes del tratamiento no puede evaluarse adecuadamente empleando la OCT-DE.

Vujosevic, et al. ${ }^{13}$, al evaluar el papel de la imagen macular estructural y funcional en la caracterización de los patrones EMD, correlacionaron mejor la AF con la microperimetría del campo central y los patrones de OCT que con la agudeza visual. Hubo una diferencia significativa en el promedio de AVMC entre tres diferentes grupos de patrones de AF (normal, unifocal y mutifocal).

En ese mismo estudio de Vujosevic, et al. ${ }^{13}$, las variables que tenían que ver con el grosor macular no fueron significativamente diferentes entre los grupos de AF. Por el contrario, en nuestro estudio, encontramos una asociación entre los diferentes patrones de AF en el caso del VM y el GPM, principalmente entre el patrón con AF disminuida y el patrón con AF normal, así como entre el patrón con AF disminuida y el patrón con AF aumentada multifocal.

En otro estudio, Vujosevic, et al..$^{10}$ evaluaron los cambios de biomarcadores de imagen retinianos específicos, como los puntos hiperreflécticos intrarretinianos, el desprendimiento neurorretiniano subfoveal y el aumento de la autofluorescencia foveal después del tratamiento con dexametasona intravítrea o ranibizumab en EMD, como posibles indicadores de la condición inflamatoria de la retina. Encontraron que el área media de AF aumentada disminuyó en ambos grupos, tanto en el de dexametasona como en el de ranibizumab.

En los ojos tratados con ranibizumab encontraron una correlación inversa significativa entre el área de AF aumentadabasaly laAVMC. Después de ambostratamientos, dexametasona y ranibizumab, el área media de AF aumentada disminuyó significativamente ${ }^{10}$.

En lo referente a las limitaciones de nuestro estudio, está el aspecto que tiene que ver con el uso de una cámara flash para obtener imágenes de AF. Las cámaras de fondo captan más luz reflejada y dispersa en comparación con los sistemas confocales. La luz dispersada desde estructuras fuera del plano retiniano puede aumentar falsamente la señal de AF, un fenómeno denominado pseudoautofluorescencia ${ }^{8}$. Por otro lado, existe un problema de variabilidad en la obtención de la señal de AF, por lo que es posible que algunos casos que por lo regular se aprecian con AF aumentada pudieran salir como de AF disminuida y viceversa. De acuerdo a Frampton, et al..$^{20}$, la sensibilidad y la especificidad del sistema basado en cámara de flash para la obtención de imágenes de AF en el EMD es variable (sensibilidad del 43 al $67 \%$ y especificidad del 86 al $99 \%)$.

Las cámaras de fondo también producen imágenes con bajo contraste, lo que puede llevar a una inadecuada interpretación de las imágenes debido a la posible confusión de los patrones de AF foveal. En este sentido, sería interesante evaluar el papel de los métodos cuantitativos de AF.

El análisis de la OCT estructural que se realizó para definir la clasificación del EMD por OCT fue solo con la valoración de la imagen del reporte en PDF del equipo que solo muestra dos cortes estructurales. Lo anterior se pudo traducir en un sesgo al clasificar de forma imprecisa los casos y, por lo tanto, la correlación se pudo ver afectada.

Se han intentado métodos para cuantificar la AF. Yoshitake, et al. ${ }^{18}$ describieron un método para cuantificar los niveles relativos de intensidad de la AF en subcampos individuales de la cuadrícula del ETDRS en EMD y evaluaron la relevancia clínica en comparación con los hallazgos del OCT. Empero, este método no es un método para el análisis cuantitativo de la AF.

Delori, et al. ${ }^{21}$ desarrollaron y probaron otro método para realizar mediciones cuantitativas estandarizadas de AF que se ha utilizado en varias patologías de la retina $^{21-24}$. Esta técnica es aplicable al escaneo de oftalmoscopia láser y, probablemente, también a cámaras de fondo de ojo.

El principio básico de este método consiste en que cuando la AF del fondo de ojo se normaliza a la fluorescencia de un estándar montado dentro del dispositivo de imagen, los efectos de la variación en la ganancia del detector y la potencia del láser se pueden compensar. Por lo tanto, la AF puede compararse longitudinalmente, entre ojos y entre imágenes obtenidas con diferentes dispositivos.

Finalmente, debido a la falta de estandarización en el equipo (principalmente por diferentes calidades de adquisición de imagen) y la falta de una base de datos normativa estandarizada para AF, la AF no es actualmente un método de examen de mucha difusión para enfermedades de la retina y del EPR.

En ninguno de los estudios sobre AF se ha examinado sistemáticamente la reproducibilidad y la consistencia 
de las mediciones de AF. Sin embargo, la AF tiene un potencial significativo como herramienta para proporcionar soluciones a muchos cuestionamientos en el área de las enfermedades maculares.

\section{Conclusiones}

En pacientes con EMD, se presentan diferentes patrones de AF con los sistemas de cámara de flash, los cuales se asocian con variables estructurales de la OCT-DE y de la FA, principalmente con el VM y GPM, así como la fuga perifoveal. En este sentido, por lo general, a mayor AF, mayor grosor promedio macular y fuga de la red capilar perifoveal por FA. Por otro lado, una clasificación fenotípica más precisa podría ayudar a determinar factores pronóstico de pérdida visual o al diseño de otros ensayos clínicos para el EMD.

La AF se erige como un estudio con importante potencial para la evaluación del EPR y más integral de la función retiniana en la era actual en donde está tan prevalente el paradigma del diagnóstico con base en la imagenología retiniana multimodal.

Por último, se requieren estudios a más largo plazo, con mayor cantidad de unidades de observación para determinar el papel más preciso de la AF en pacientes con EMD.

\section{Conflicto de intereses}

Los autores declaran no tener intereses financieros en la realización de este estudio.

\section{Responsabilidades éticas}

Protección de personas y animales. Los autores declaran que los procedimientos seguidos se conformaron a las normas éticas del comité de experimentación humana responsable y de acuerdo con la Asociación Médica Mundial y la Declaración de Helsinki.

Confidencialidad de los datos. Los autores declaran que han seguido los protocolos de su centro de trabajo sobre la publicación de datos de pacientes.

Derecho a la privacidad y consentimiento informado. Los autores han obtenido el consentimiento informado de los pacientes y/o sujetos referidos en el artículo. Este documento obra en poder del autor de correspondencia.

\section{Bibliografía}

1. Klein R, Knudtson MD, Lee KE, Gangnon R, Klein BE. The Wisconsin Epidemiologic Study of Diabetic Retinopathy XXIII: twenty-five-year incidence of macular edema in persons with type 1 diabetes. Ophthalmology. 2009;116:497-503.

2. Girach $A$, Lund-Andersen $H$. Diabetic macular oedema: a clinical overview. Int J Clin Pract. 2007;61:88-97.

3. Shaw JE, Sicree RA, Zimmet PZ. Global estimates of the prevalence of diabetes for 2010 and 2030. Diabetes Res Clin Pract. 2010; 87:4-14.

4. Velasco-Guzmán BJ, Brena-Ramos VM. DM tipo 2: Epidemiología y Emergencia en Salud. Salud y Administración. 2014;1:11-6.

5. Acon D, Wu L. Multimodal Imaging in Diabetic Macular Edema. Asia Pac J Ophthalmol (Phila). 2018;7:22-7.

6. Midena E, Bini S. Multimodal retinal imaging of diabetic macular edema: toward new paradigms of pathophysiology. Graefes Arch Clin Exp Ophthalmol. 2016;254:1661-8.

7. Pichi F, Abboud EB, Ghazi NG, Khan AO. Fundus autofluorescence imaging in hereditary retinal diseases. Acta Ophthalmol. 2018;96: e549-61.

8. Yung M, Klufas MA, Sarraf D. Clinical applications of fundus autofluorescence in retinal disease. Int J Retina Vitreous. 2016;2:12.

9. McBain VA, Forrester JV, Lois N. Fundus autofluorescence in the diagnosis of cystoid macular oedema. Br J Ophthalmol. 2008;92:946-9.

10. Vujosevic S, Torresin T, Bini S, Convento E, Pilotto E, Parrozzani R, et al. Imaging retinal inflammatory biomarkers after intravitreal steroid and anti-VEGF treatment in diabetic macular oedema. Acta Ophthalmol. 2017;95:464-71.

11. Chung H, Park B, Shin HJ, Kim HC. Correlation of fundus autofluorescence with spectral-domain optical coherence tomography and vision in diabetic macular edema. Ophthalmology. 2012;119:1056-65.

12. Vujosevic S, Bottega E, Casciano M, Pilotto E, Convento E, Midena E. Microperimetry and fundus autofluorescence in diabetic macular edema: subthreshold micropulse diode laser versus modified early treatment diabetic retinopathy study laser photocoagulation. Retina. 2010; 30:908-16.

13. Vujosevic S, Casciano M, Pilotto E, Boccassini B, Varano M, Midena E. Diabetic macular edema: fundus autofluorescence and functional correlations. Invest Ophthalmol Vis Sci. 2011;52:442-8.

14. Shen $Y, X u X$, Liu K. Fundus autofluorescence characteristics in patients with diabetic macular edema. Chin Med J. 2014;127:1423-8.

15. Reznicek L, Dabov S, Haritoglou C, Kampik A, Kernt M, Neubauer AS Green-light fundus autofluorescence in diabetic macular edema. Int $J$ Ophthalmol. 2013;6:75-80.

16. Pece A, Isola V, Holz F, Milani P, Brancato R. Autofluorescence imaging of cystoid macular edema in diabetic retinopathy. Ophthalmologica. 2010;224:230-5

17. Otani T, Kishi S, Maruyama Y. Patterns of diabetic macular edema with optical coherence tomography. Am J Ophthalmol. 1999;127:688-93.

18. Yoshitake S, Murakami T, Uji A, Unoki N, Dodo Y, Horii T, et al. Clinical relevance of quantified fundus autofluorescence in diabetic macular oedema. Eye. 2015;29:662-9.

19. Calvo-Maroto AM, Perez-Cambrodi RJ, Garcia-Lazaro S, Ferrer-Blasco T, Cerviño A. Ocular autofluorescence in DM. A review. JDM. 2016;8:619-28.

20. Frampton GK, Kalita N, Payne L, Colquitt JL, Loveman E, Downes SM, Lotery AJ. Fundus autofluorescence imaging: systematic review of test accuracy for the diagnosis and monitoring of retinal conditions. Eye (Lond). 2017;31:995-1007.

21. Delori F, Greenberg JP, Woods RL, Fischer J, Duncker T, Sparrow J, Smith RT. Quantitative measurements of autofluorescence with the scanning laser ophthalmoscope. Invest Ophthalmol Vis Sci. 2011; 52:9379-90.

22. Quantitative fundus autofluorescence in healthy eyes. Greenberg JP, Duncker T, Woods RL, Smith RT, Sparrow JR, Delori FC. Invest Ophthalmol Vis Sci. 2013;54:5684-93.

23. Marsiglia M, Lee W, Mahajan VB, Zernant J, Delori FC, Tsang SH, Sparrow JR. Quantitative autofluorescence as a clinical tool for expedited differential diagnosis of retinal degeneration. JAMA Ophthalmol. 2015; 133:219-20.

24. Duncker T, Tsang SH, Lee W, Zernant J, Allikmets R, Delori FC, Sparrow JR. Quantitative fundus autofluorescence distinguishes ABCA4-associated and nonAB CA4-associated bull's-eye maculopathy. Ophthalmology. 2015;122:345-55. 